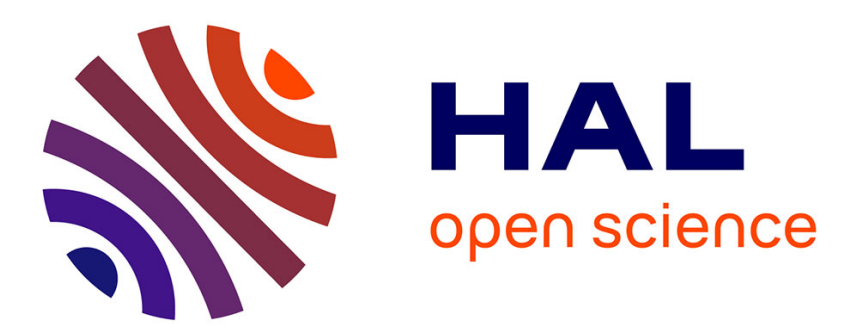

\title{
Current-based Analytical Model Derivation to Analyse fault effects in 5-phase PMSM
}

Demba Diallo, Claude Delpha

\section{To cite this version:}

Demba Diallo, Claude Delpha. Current-based Analytical Model Derivation to Analyse fault effects in 5-phase PMSM. IEEE International Power Electronics and Application Conference and Exposition (IEEE PEAC'2018), Nov 2018, Shenzhen, China. 10.1109/peac.2018.8590319 . hal-01895233

\section{HAL Id: hal-01895233 \\ https://hal-centralesupelec.archives-ouvertes.fr/hal-01895233}

Submitted on 12 Mar 2020

HAL is a multi-disciplinary open access archive for the deposit and dissemination of scientific research documents, whether they are published or not. The documents may come from teaching and research institutions in France or abroad, or from public or private research centers.
L'archive ouverte pluridisciplinaire HAL, est destinée au dépôt et à la diffusion de documents scientifiques de niveau recherche, publiés ou non, émanant des établissements d'enseignement et de recherche français ou étrangers, des laboratoires publics ou privés. 


\section{Current-Based Analytical Model Derivation to Analyse Fault Effects in 5-Phase PMSM}

\author{
D. Diallo, IEEE Senior Member \\ Group of Electrical Engineering of Paris \\ CNRS UMR 8507, CentraleSupelec, Univ. Paris Sud, Université \\ Paris Saclay, \\ Sorbonne Université \\ Gif Sur Yvette, France \\ demba.diallo@geeps.centralesupelec.fr
}

\author{
C. Delpha, IEEE Senior Member \\ Laboratory of Signals and Systems \\ CNRS UMR 8506, CentraleSupelec, Univ. Paris Sud, Université \\ Paris Saclay \\ Gif Sur Yvette, France \\ claude.delpha@12s.centralesupelec.fr
}

\begin{abstract}
In this paper, we present an analytical model of the currents flowing in a 5-phase PMSM. The analytical expressions of the currents are derived both in the stationary and the synchronous frames. This analytical model depends on the fault effect parameters: phase shift, amplitude modification and bias. The flexibility of the model allows analysing the different fault signatures in any of the reference frames whatever the coupling of the windings (isolated or non isolated neutral). At first, this analytical model has been validated by comparison with a numerical model obtained from simulations. Afterwards it has been used to evaluate the fault effect on the transformed currents. The results have shown that depending on the fault type, the current waveforms exhibit changes (additional components, mean or variance changes) and the current vector trajectory is also distorted. Therefore, the fault signature may be extracted from features in the time, frequency or time-frequency domain. The results have also shown that the fault features should be tracked in all the frames.
\end{abstract}

Keywords - 5-phase permanent magnet synchronous machine, fault modelling, fault diagnosis

\section{INTRODUCTION}

Multiphase machines (ie. with number of phases higher than 3 ) have regained attention in the last decades [1]. The higher number of phases facilitates the power splitting and naturally increases the resilience. They are attractive in high power applications or in applications with high requirements of availability. This is the case for aerospace and marine applications [2-4]. Electrical machines are more and more present in ship propulsion or on-board electrical energy production. One of the most popular multiphase machines is the five-phase permanent magnet synchronous machine [5]. For maritime applications, the design is conservative because no breakdown is tolerable. Moreover the duration of stopping ships for maintenance must be minimal due to the high cost. Therefore it is relevant to develop efficient maintenance tools to increase the reliability and the availability. Condition-based maintenance is now widely accepted as the most suitable method. It requires continuous monitoring and the implementation of Fault Detection and Diagnosis (FDD) methods [6-8]. In electrified ships, the currents flowing in the machine windings contain valuable information on the status of the electrical actuator.

Following the general scheme described in Fig. 1 for each of the four steps, different approaches can be used.

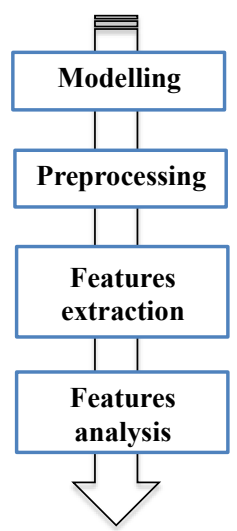

Fig. 1. Fault Detection and Diagnosis general scheme

The requirements for a fault detection and diagnosis method are summarized in Fig. 2 [9].

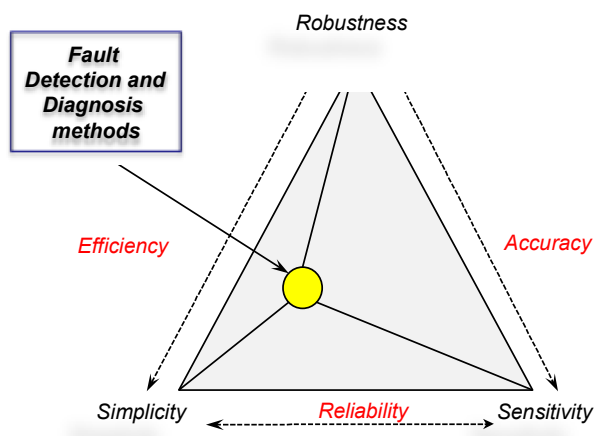

Fig. 2. Fault Detection and Diagnosis method requirements

They are defined as follows:

- The simplicity that is defined as the amount of information for processing;

- The sensitivity that is defined as the capability of the method to detect a fault at its earliest stage;

- The robustness that measures the capability of the method to perform despite the perturbations.

The selection of a method is a compromise between these requirements.

In this paper, we are interested in developing an analytical model that can be used for a systematic analysis of the fault effect in a 5-phase PMSM. The model is based on the currents flowing in the machine windings. The main assumption is that any fault in the drive (in the power 
converter, the machine or the load) will affect the current. Therefore, fault features should be retained from the current vector trajectory or characteristics in a stationary or synchronous frame.

The paper is organised as follows. In section II the fault effect modelling is presented. The expressions of the currents in the different frames are derived. In section III, the analyses of the effects for the different fault types are presented. Finally, a conclusion closes the paper.

\section{FAULT EFFECT MODELLING}

\section{A- Phase Current modelling}

The 5-phase Permanent Magnet Synchronous Machine can be modelled in three different frames:

○ the natural frame with the 5 phases for which the variables are denoted $x_{1}$ to $x_{5}$,

○ the stationary transformed frame with 3 equivalent fictitious machines (a one-phase homopolar machine for which the variables are denoted $x_{0}$, a two-phase principal machine for which the variables are denoted $x_{p \alpha}$ and $x_{p}$, and another two-phase secondary machine for which the variables are denoted $x_{s} \alpha$ and $x_{s} \beta$

o the rotating transformed frame with also 3 equivalent fictitious machines (a one-phase homopolar machine for which the variables are denoted $x_{O_{S}}$, a two-phase principal machine for which the variables are denoted $x_{p d}$ and $x_{p q}$, and a two-phase secondary machine for which the variables are denoted $x_{s d}$ and $x_{s q}$.

We assume that any fault in the energy conversion chain (power converter, electrical machine or sensors) will affect the currents. The fault will induce a modification of the amplitude (gain fault $\Delta i_{j}$ ), the mean value $\left(\gamma_{j}\right)$ or a phase shift $\left(\phi_{j}\right)$ in the $\mathrm{j}^{\text {th }}$ phase.

In the following, we limit our study to the fundamental component of the phase currents.

We can therefore write the faulty current in the natural frame as:

$$
i_{j(j=1 . .5)_{f}}=I \sqrt{2}\left(1+\Delta i_{j}\right) \sin \left(\theta+(1-j) \frac{2 \pi}{5}+\phi_{j}\right)+\gamma_{j}
$$

Where $\theta=\omega t, \omega$ is the angular frequency, $\mathrm{t}$ the current time and $I$ represents the RMS value of the phase current.

In the following we will adopt the simplified following notation:

$$
\delta_{j}=I \sqrt{2}\left(1+\Delta i_{j}\right)
$$

The transform matrixes from the natural frame to the stationary frame and from the stationary frame to the rotating one are respectively denoted $T_{n s}$ and $R_{s r}$ and are defined as:

$$
T_{n s}=\sqrt{\frac{2}{5}}\left[\begin{array}{ccccc}
\frac{1}{\sqrt{2}} & \frac{1}{\sqrt{2}} & \frac{1}{\sqrt{2}} & \frac{1}{\sqrt{2}} & \frac{1}{\sqrt{2}} \\
1 & \cos \left(\frac{2 \pi}{5}\right) & \cos \left(\frac{4 \pi}{5}\right) & \cos \left(\frac{6 \pi}{5}\right) & \cos \left(\frac{8 \pi}{5}\right) \\
0 & \sin \left(\frac{2 \pi}{5}\right) & \sin \left(\frac{4 \pi}{5}\right) & \sin \left(\frac{6 \pi}{5}\right) & \sin \left(\frac{8 \pi}{5}\right) \\
1 & \cos \left(\frac{4 \pi}{5}\right) & \cos \left(\frac{8 \pi}{5}\right) & \cos \left(\frac{12 \pi}{5}\right) & \cos \left(\frac{16 \pi}{5}\right) \\
0 & \sin \left(\frac{4 \pi}{5}\right) & \sin \left(\frac{8 \pi}{5}\right) & \sin \left(\frac{12 \pi}{5}\right) & \sin \left(\frac{16 \pi}{5}\right)
\end{array}\right]
$$

and

$$
R_{s r}=\left[\begin{array}{ccccc}
1 & 0 & 0 & 0 & 0 \\
0 & \cos (\theta) & -\sin (\theta) & 0 & 0 \\
0 & \sin (\theta) & \cos (\theta) & 0 & 0 \\
0 & 0 & 0 & \cos (3 \theta) & \sin (3 \theta) \\
0 & 0 & 0 & -\sin (3 \theta) & \cos (3 \theta)
\end{array}\right]
$$

With these matrixes, the transformed currents can be obtained as follows in the stationary frame:

$$
\left[\begin{array}{c}
x_{0} \\
x_{p \alpha} \\
x_{p \beta} \\
x_{s \alpha} \\
x_{s \beta}
\end{array}\right]=\sqrt{\frac{2}{5}}\left[\begin{array}{ccccc}
\frac{1}{\sqrt{2}} & \frac{1}{\sqrt{2}} & \frac{1}{\sqrt{2}} & \frac{1}{\sqrt{2}} & \frac{1}{\sqrt{2}} \\
1 & \cos \left(\frac{2 \pi}{5}\right) & \cos \left(\frac{4 \pi}{5}\right) & \cos \left(\frac{6 \pi}{5}\right) & \cos \left(\frac{8 \pi}{5}\right) \\
0 & \sin \left(\frac{2 \pi}{5}\right) & \sin \left(\frac{4 \pi}{5}\right) & \sin \left(\frac{6 \pi}{5}\right) & \sin \left(\frac{8 \pi}{5}\right) \\
1 & \cos \left(\frac{4 \pi}{5}\right) & \cos \left(\frac{8 \pi}{5}\right) & \cos \left(\frac{12 \pi}{5}\right) & \cos \left(\frac{16 \pi}{5}\right) \\
0 & \sin \left(\frac{4 \pi}{5}\right) & \sin \left(\frac{8 \pi}{5}\right) & \sin \left(\frac{12 \pi}{5}\right) & \sin \left(\frac{16 \pi}{5}\right)
\end{array}\right]\left[\begin{array}{c}
x_{1} \\
x_{2} \\
x_{3} \\
x_{4} \\
x_{5}
\end{array}\right]
$$

and in the synchronous rotating one:

$\left[\begin{array}{c}x_{0 s s} \\ x_{p d} \\ x_{p q} \\ x_{s d} \\ x_{s q}\end{array}\right]=\left[\begin{array}{ccccc}1 & 0 & 0 & 0 & 0 \\ 0 & \cos (\theta) & -\sin (\theta) & 0 & 0 \\ 0 & \sin (\theta) & \cos (\theta) & 0 & 0 \\ 0 & 0 & 0 & \cos (3 \theta) & \sin (3 \theta) \\ 0 & 0 & 0 & -\sin (3 \theta) & \cos (3 \theta)\end{array}\right]\left[\begin{array}{c}x_{h} \\ x_{p \alpha} \\ x_{p \beta} \\ x_{s \alpha} \\ x_{s \beta}\end{array}\right]$

The results obtained with two transformations will be denoted as simulated variables.

When a fault occurs, the currents flowing in the windings are distorted, so will be the transformed ones. However, the analysis of the fault (type and level) on the currents would be improved if we have at our disposal an analytical model of the transformed currents [10-11]. This model should depend on the fault parameters and be as accurate as the numerical model derived from the transformation matrixes. But it will be more flexible as it will offer the possibility to analyse the waveforms to detect the fault but also to estimate the fault severity and enhance fault isolation.

\section{B- Modelling of faulty current in the stationary frame}

Notation: in the following, variables with lower script " $h$ " refer to healthy condition while those with lower script "f" refer to the faulty case.

From (1) and using the matrix $T_{n s}$, we can derive the analytical expressions of the currents in the transformed stationary frame as follows: 


$$
\begin{aligned}
& x_{p \alpha_{f}}=\frac{2}{\sqrt{5}}(A \sin (\theta)+B \cos (\theta)+C) \\
& x_{p \beta_{f}}=\frac{2}{\sqrt{5}}(D \sin (\theta)+E \cos (\theta)+F) \\
& x_{s \alpha_{f}}=\frac{2}{\sqrt{5}}(G \sin (\theta)+H \cos (\theta)+J) \\
& x_{s \beta_{f}}=\frac{2}{\sqrt{5}}(K \sin (\theta)+L \cos (\theta)+M) \\
& x_{0_{f}}=\frac{1}{\sqrt{5}}(N \sin (\theta)+O \cos (\theta)+P)
\end{aligned}
$$

Where the coefficients depend on fault parameters.

$$
\begin{aligned}
& A=\delta_{1} \cos \left(\phi_{1}\right)+\frac{1}{2} \sum_{j=2}^{5} \delta_{j}\left(\cos \left(\phi_{j}\right)+\cos \left(\phi_{j}-(j-1) \frac{4 \pi}{5}\right)\right) \\
& B=\delta_{1} \sin \left(\phi_{1}\right)+\frac{1}{2} \sum_{j=2}^{5} \delta_{j}\left(\sin \left(\phi_{j}\right)+\sin \left(\phi_{j}-(j-1) \frac{4 \pi}{5}\right)\right) \\
& C=\gamma_{1}+\gamma_{2} \cos \left(\frac{2 \pi}{5}\right)+\gamma_{3} \cos \left(\frac{4 \pi}{5}\right)+\gamma_{4} \cos \left(\frac{6 \pi}{5}\right)+\gamma_{5} \cos \left(\frac{8 \pi}{5}\right) \\
& D=\frac{1}{2} \sum_{j=2}^{5} \delta_{j}\left(\sin \left(\phi_{j}\right)-\sin \left(\phi_{j}-(j-1) \frac{4 \pi}{5}\right)\right) \\
& E=\frac{1}{2} \sum_{j=2}^{5} \delta_{j}\left(-\cos \left(\phi_{j}\right)+\cos \left(\phi_{j}-(j-1) \frac{4 \pi}{5}\right)\right) \\
& F=\gamma_{2} \sin \left(\frac{2 \pi}{5}\right)+\gamma_{3} \sin \left(\frac{4 \pi}{5}\right)+\gamma_{4} \sin \left(\frac{6 \pi}{5}\right)+\gamma_{5} \sin \left(\frac{8 \pi}{5}\right) \\
& G=\delta_{1} \cos \left(\phi_{1}\right)+\sum_{j=2}^{5} \delta_{j} \cos \left(\frac{4 \pi(j-1)}{5}\right) \cos \left(\phi_{j}-(j-1) \frac{2 \pi}{5}\right) \\
& H=\delta_{1} \sin \left(\phi_{1}\right)+\sum_{j=2}^{5} \delta_{j} \cos \left(\frac{4 \pi(j-1)}{5}\right) \sin \left(\phi_{j}-(j-1) \frac{2 \pi}{5}\right) \\
& J=\gamma_{1}+\gamma_{2} \cos \left(\frac{4 \pi}{5}\right)+\gamma_{3} \cos \left(\frac{8 \pi}{5}\right)+\gamma_{4} \cos \left(\frac{2 \pi}{5}\right)+\gamma_{5} \cos \left(\frac{6 \pi}{5}\right) \\
& K=\sum_{j=2}^{5} \delta_{j} \sin \left(\frac{4 \pi(j-1)}{5}\right) \cos \left(\phi_{j}-(j-1) \frac{2 \pi}{5}\right) \\
& L=\sum_{j=2}^{5} \delta_{j} \sin \left(\frac{4 \pi(j-1)}{5}\right) \sin \left(\phi_{j}-(j-1) \frac{2 \pi}{5}\right) \\
& M=\gamma_{1}+\gamma_{2} \sin \left(\frac{4 \pi}{5}\right)+\gamma_{3} \sin \left(\frac{8 \pi}{5}\right)+\gamma_{4} \sin \left(\frac{2 \pi}{5}\right)+\gamma_{5} \sin \left(\frac{6 \pi}{5}\right)
\end{aligned}
$$

and

$$
\begin{aligned}
N & =\sum_{j=1}^{5} \delta_{j} \cos \left(\phi_{j}-(j-1) \frac{2 \pi}{5}\right) \\
O & =\sum_{j=1}^{5} \delta_{j} \sin \left(\phi_{j}-(j-1) \frac{2 \pi}{5}\right) \\
P & =\sum_{j=1}^{5} \gamma_{j}
\end{aligned}
$$

As one can see in Fig. 3, for the case of a combined fault (gain, phase shift and offset) the analytical model fits with the numerical one obtained from simulations. It can be therefore used for the analysis of fault effect and the extraction of fault signatures.

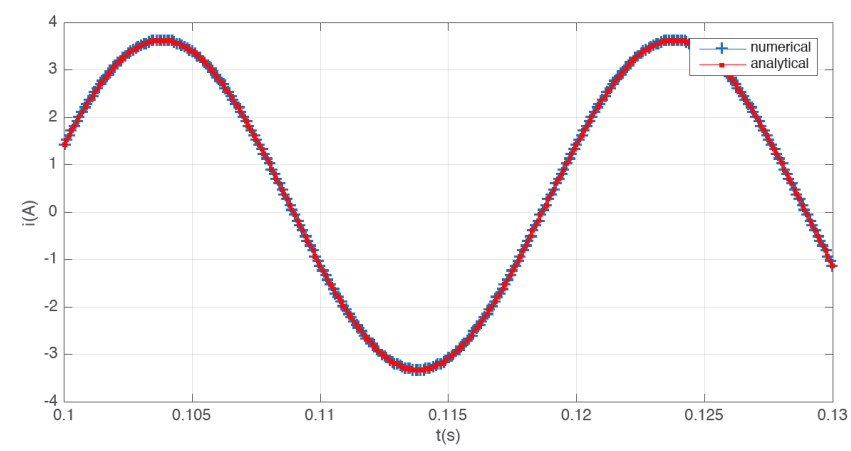

Fig. 3. Phase currents flowing in the fictitious primary machine

\section{C- Modelling of faulty current in the synchronous frame}

In the same way, the expressions of the currents in the synchronous rotating frame can be derived from (2) and the rotation matrix $R_{s r}$.

$x_{p d_{f}}=\frac{2}{\sqrt{5}}\left(\frac{1}{2}(A+E) \sin (2 \theta)+\frac{1}{2}(B+D) \cos (2 \theta)+C \cos (\theta)-F \sin (\theta)+\frac{1}{2}(B-D)\right)$

$x_{p q_{f}}=\frac{2}{\sqrt{5}}\left(\frac{1}{2}(B+D) \sin (2 \theta)+\frac{1}{2}(E+A) \cos (2 \theta)+C \sin (\theta)+F \cos (\theta)+\frac{1}{2}(-A+E)\right)$

$x_{s d_{f}}=\frac{2}{\sqrt{5}}\left(\frac{1}{4}(2 L+G) \sin (4 \theta)+\frac{1}{4}(2 H-2 K-G) \cos (4 \theta)\right.$

$\left.+M \sin (3 \theta)+J \cos (3 \theta)+\frac{1}{2}(L-G) \sin (2 \theta)+\frac{1}{2}(H+K) \cos (2 \theta)+\frac{G}{4}\right)$

$x_{s q_{f}}=\frac{2}{\sqrt{5}}\left(\frac{1}{4}(2 K-H-L) \sin (4 \theta)+\frac{1}{4}(2 L+G) \cos (4 \theta)+\right.$

$\left.-J \sin (3 \theta)+M \cos (3 \theta)-\frac{1}{2}(H+K) \sin (2 \theta)+\frac{1}{2}(L-G) \cos (2 \theta)+\frac{L}{4}\right)$

$x_{s 0_{f}}=x_{0 f}$

Through the coefficients defined in (3), (4), (5) and (6), these currents also depend on the fault parameters.

As one can see in Fig. 4, for the case of a combined fault (gain, phase shift and offset) the analytical model in the synchronous frame also fits with the numerical one. It can be therefore used for the analysis of fault effect and the extraction of fault signatures.

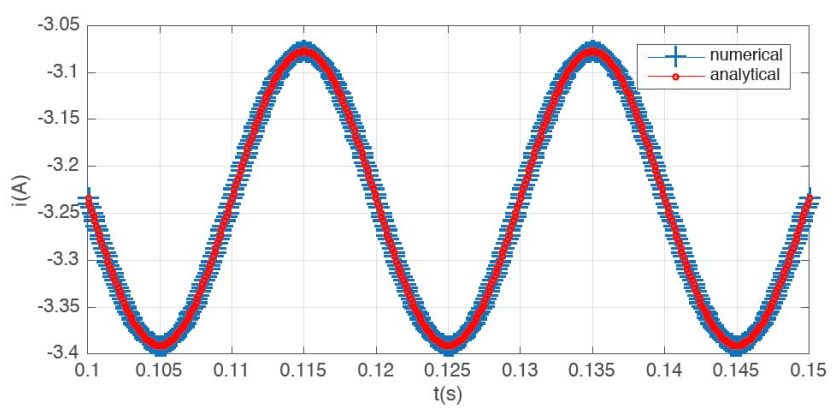

Fig. 4. $\mathrm{q}$ axis currents flowing in the fictitious primary machine

\section{FAULT EFFECT ANALYSIS}

In healthy condition, in the stationary frame there is no current flowing in the secondary machine and no homopolar current as confirmed by the following equation: 
$x_{p \alpha_{h}}=2 \sqrt{5} I \sin (\theta)$

$x_{p \beta_{h}}=2 \sqrt{5} I \sin \left(\theta-\frac{\pi}{2}\right)$

$x_{s \alpha_{h}}=0, x_{s \beta h}=0$

$x_{0_{h}}=0$

And in the synchronous frame, the currents are of DC type and only one component is not null as confirmed in (10).

$x_{p d_{h}}=0$

$x_{p q h}=-\sqrt{10} I$

$x_{s d_{h}}=0, x_{s q_{h}}=0$

$x_{s 0_{h}}=0$

At fault occurrence, new components and distortions will appear in these current components. The fault diagnosis method should be able to extract significant and sensitive features from the waveforms or from the current vector trajectory.

However, to use properly this model, the coupling mode of the phases should be known. Because depending on how the windings of the machine are connected, e.g. isolated or non isolated neutral, the model is constrained and the fault parameters should be tuned adequately.

\section{A. Isolated Neutral}

If the neutral of the machine is isolated or ground-connected with high impedance, the phase currents are constrained such as:

$\forall t>0$

$\sum_{j=1}^{5} i_{j}=\sum_{j=1}^{5} I \sqrt{2}\left(1+\Delta i_{j}\right) \sin \left(\theta+(1-j) \frac{2 \pi}{5}+\phi_{j}\right)+\gamma_{j}=0$

As a consequence, to evaluate a single gain fault in phase 1 (meaning no offset nor phase shift), setting $\Delta i_{1} \neq 0$ would not be sufficient to satisfy (9). To evaluate a gain fault in one phase of isolated neutral machine, the same fault level should be set in each phase as: $\Delta i_{1}=\Delta i_{2}=\Delta i_{3}=\Delta i_{4}=\Delta i_{5}$.

One can see the effect of a $10 \%$ gain fault in one phase on the current trajectory in the stationary frame $(\alpha, \beta)$ of the primary fictitious machine in Fig. 5.

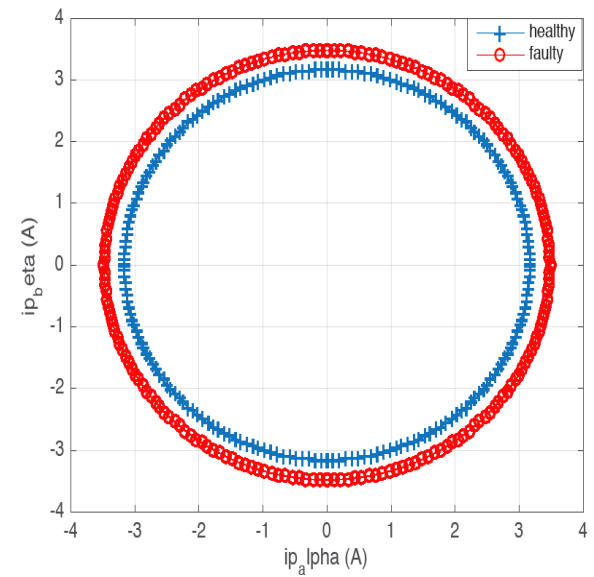

Fig. 5. Trajectory of the current vector in the stationary frame of the primary fictitious machine ( $10 \%$ gain fault in phase A)

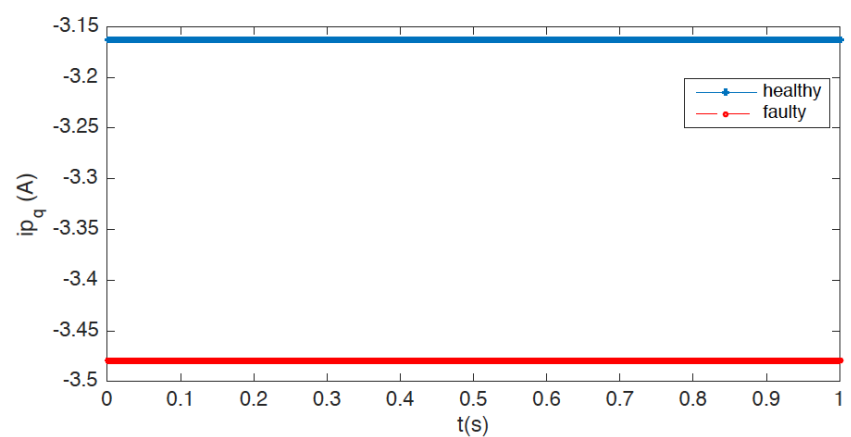

Fig. 6. Fictitious primary machine q axis currents in the synchronous frame $(10 \%$ gain fault in phase $\mathrm{A})$

If we observe the results displayed in Figs. 5 and 6, it can be concluded that the fault features should be looked for in the variation of the variance in the primary fictitious machine components in the stationary reference frame or in the mean value change of the primary fictitious machine components in the synchronous reference frame. These parameters can be considered as fault features [12].

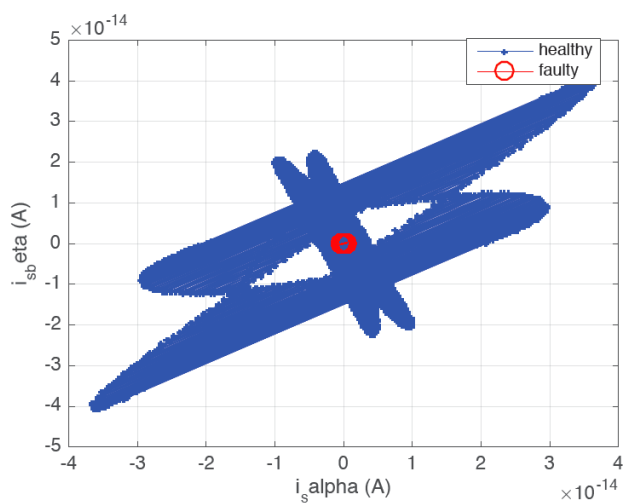

Fig. 7. Trajectory of the current vector in the stationary frame of the secondary fictitious machine (10\% gain fault in phase A)

However, if we look at Fig. 7, there is clearly no fault effect in the stationary frame $(\alpha, \beta)$ of the secondary fictitious machine.

\section{B. Non-Isolated Neutral}

If the neutral of the machine is connected, (11) is no longer respected, as there is a homopolar current component.

In this case for a for a $10 \%$ gain fault in phase $A$, we should set, $\Delta i_{1}=0.1$ and $\Delta i_{2}=\Delta i_{3}=\Delta i_{4}=\Delta i_{5}=0$. If we observe the current trajectory of the primary fictitious machine in the stationary frame in Fig. 8, we can notice the distortion of the trajectory from a circle to an ellipse. The parameters of this new trajectory can be used as fault features.

Compared with Fig. 5, we can also notice the effect of the phase coupling despite the same fault conditions. 


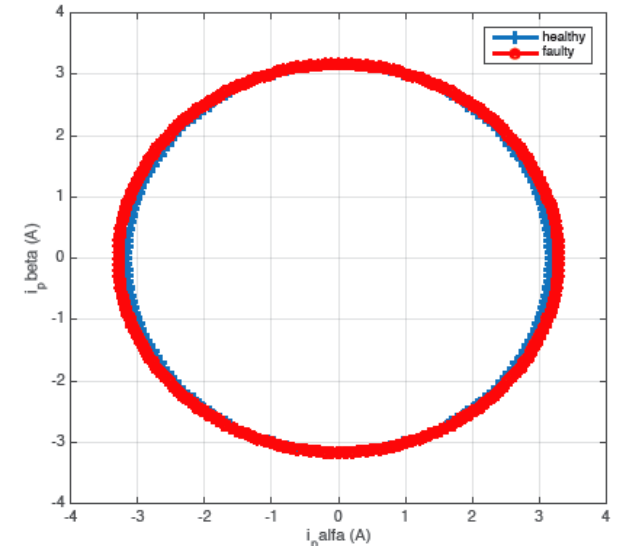

Fig. 8. Trajectory of the current vector in the stationary frame $(\alpha, \beta)$ of the primary fictitious machine ( $10 \%$ gain fault in phase A)

If we look at Fig. 9 and compare it with the results displayed in Fig. 6, we can also notice the appearance of a current component flowing in the $\alpha$ axis of the secondary fictitious machine. The characteristics of this current can be in this case used as fault features.

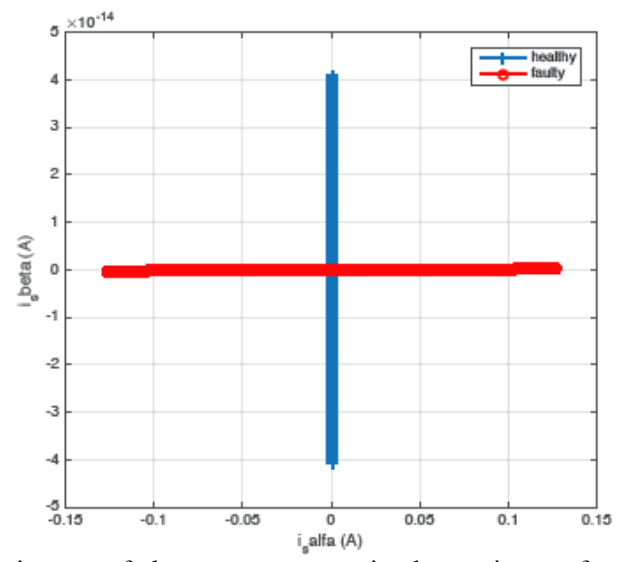

Fig. 9. Trajectory of the current vector in the stationary frame of the secondary fictitious machine ( $10 \%$ gain fault in phase A)

Compared with Fig. 6, if the neutral is not isolated, for the same fault conditions, if we look at Fig. 10, we can see that in both $d$ and $q$ axes, the characteristics of the currents differ significantly from the healthy case.

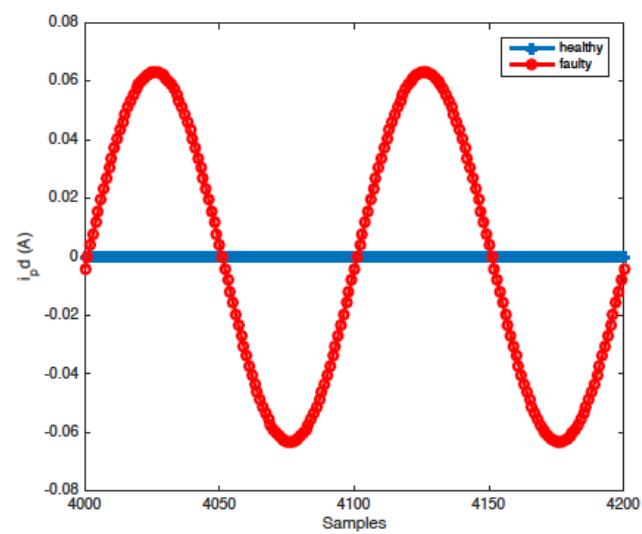

a) d axis currents

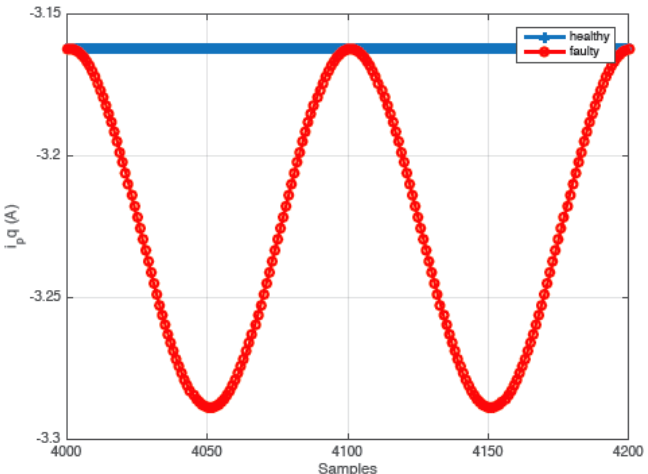

b) q axis currents

Fig. 10. $(d, q)$ fictitious primary machine currents in the synchronous frame $(10 \%$ gain fault in phase $\mathrm{A})$

In the case of a $30 \%$ gain fault in phase $\mathrm{C}$ we should set, $\Delta i_{3}=0.3$ and $\Delta i_{1}=\Delta i_{2}=\Delta i_{4}=\Delta i_{5}=0$. If we observe the current trajectory of the primary fictitious machine in the stationary frame displayed in Fig. 11, we can notice both a phase shift and the distortion of the trajectory from a circle to an ellipse. These parameters can be used as fault features.

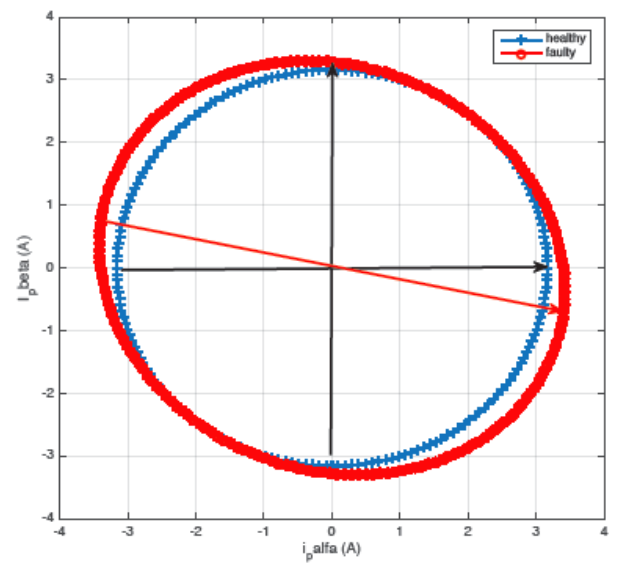

Fig. 11. Trajectory of the current vector in the stationary frame $(\alpha, \beta)$ of the primary fictitious machine (30\% gain fault in phase C)

In the case of a combined fault (gain, offset and phase shift) in phase $\mathrm{C}$, the trajectory of the current in the fictitious primary machine is displayed in Fig. 12. One can also notice a distortion of the trajectory with a simultaneous modification of the amplitude and a phase shift. 


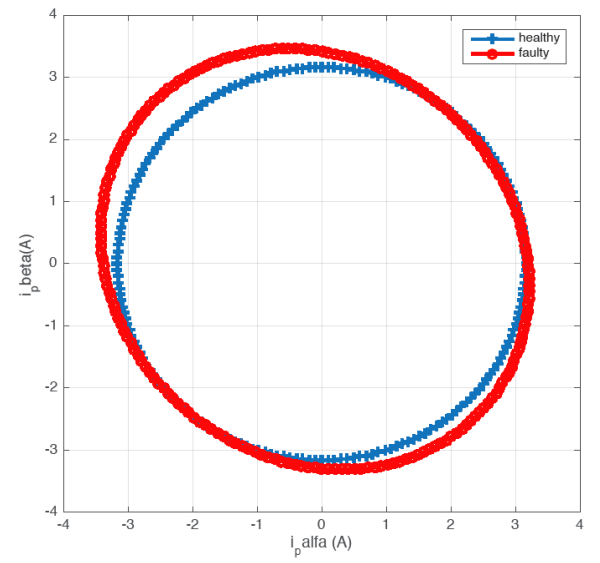

Fig. 12. Trajectory of the current vector in the stationary frame $(\alpha, \beta)$ of the primary fictitious machine (30\% gain fault, $10 \%$ phase shift and $10 \%$ offset fault)

For the same fault conditions, if we look at the waveforms displayed in Fig. 13, we can also notice significant changes from the healthy case with additional components combined with mean value and variance changes.

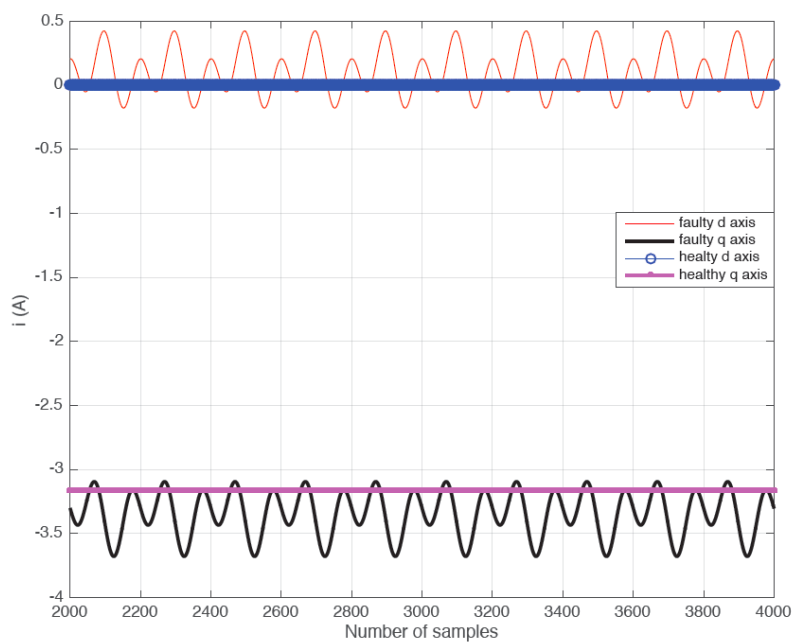

Fig. 13. Primary fictitious machine $(d, q)$ current waveforms in the synchronous frame (30\% gain fault, 10\% phase shift and 10\% offset fault)

\section{CONCLUSION}

In this paper, we have presented an analytical model of the currents flowing in a 5-phase PMSM. This analytical model has been validated with the numerical model obtained with simulations. This analytical model is devoted to design the fault diagnosis methodology. The main assumption of this model is that whatever the fault, there is a signature in the currents flowing in the windings. The fault can induce a phase shift or/and a modification of the amplitude or/and a bias. The parameters of this analytical model are all related to the fault effect. Therefore, the model is flexible and allows analysing all the fault signatures in all the frames (stationary and synchronous). The results have shown that depending on the fault type, its signature may be extracted from features in the time, frequency or time-frequency domain. The results have also shown that the fault features should be also examined in all the frames.

It should also be pointed out that the model could be extended to cope with environmental nuisances so as to develop the most efficient fault detection, fault isolation and fault estimation methodology.

\section{REFERENCES}

[1] E. Levi, "Multiphase electric machines for variable-speed applications", IEEE Trans. Ind. Electron., vol. 55, no. 5, pp. 1893-1909, May 2008.

[2] M. Qiao, C. Jiang, Y. Zhu and G. Li, "Research on Design Method and Electromagnetic Vibration of Six-Phase Fractional-Slot ConcentratedWinding PM Motor Suitable for Ship Propulsion," in IEEE Access, vol. 4, pp. 8535-8543, 2016.

[3] K. Nounou, J. F. Charpentier, K. Marouani, M. Benbouzid and A. Kheloui, "Hardware-in-the-Loop Emulation of an Electric Naval Propulsion System Based on a Multiphase Permanent Magnet Synchronous Machine," 2017 IEEE Vehicle Power and Propulsion Conference (VPPC), Belfort, 2017, pp. 1-6.

[4] Z. Liu, J. Wu and L. Hao, "Coordinated and fault-tolerant control of tandem 15-phase induction motors in ship propulsion system," in IET Electric Power Applications, vol. 12, no. 1, pp. 91-97, 12018.

[5] X. Xue, W. Zhao, J. Zhu, G. Liu, X. Zhu and M. Cheng, "Design of Five-Phase Modular Flux-Switching Permanent-Magnet Machines for High Reliability Applications," in IEEE Transactions on Magnetics, vol. 49, no. 7, pp. 3941-3944, July 2013.

[6] A. Mohammadpour, S. Sadeghi and L. Parsa, "A Generalized FaultTolerant Control Strategy for Five-Phase PM Motor Drives Considering Star, Pentagon, and Pentacle Connections of Stator Windings," in IEEE Transactions on Industrial Electronics, vol. 61, no. 1, pp. 63-75, Jan. 2014.

[7] A. Arafat, S. Choi and J. Baek, "Open-Phase Fault Detection of a FivePhase Permanent Magnet Assisted Synchronous Reluctance Motor Based on Symmetrical Components Theory," in IEEE Transactions on Industrial Electronics, vol. 64, no. 8, pp. 6465-6474, Aug. 2017.

[8] M. Trabelsi, N. Ky Nguyen, E. Semail, "Real-Time Switches Fault Diagnosis Based on Typical Operating Characteristics of Five-Phase Permanent-Magnetic Synchronous Machines", Industrial Electronics IEEE Transactions on, vol. 63, pp. 4683-4694

[9] C. Delpha, D. Diallo, "Incipient Fault Detection and Diagnosis: a Hidden Information Detection Problem," IEEE ISIE 2015, Conference Proceedings, Buzios, Rio de Janeiro, Brazil

[10] C. Delpha, D. Diallo, H. Al Samrout, N. Moubayed "Multiple incipient fault diagnosis in three-phase electrical systems using multivariate statistical signal processing," Elsevier, Engineering Applications of Artificial Intelligence, Volume 73, August 2018, Pages 68-79

[11] C. Delpha, D. Diallo, H. Al Samrout, N. Moubayed "Analytical Model of Multiple Fault Effect in Three Phases Electrical Systems," IEEE IECON 2016, Conference Proceedings, Florence, Italy

[12] M. Basseville, V. I. Nikiforov; Detection of Abrupt Changes - Theory and Application, published by Prentice-Hall, Inc. (ISBN 0-13-126780-9 April 1993 - Englewood Cliffs, N.J.) 